\title{
Tunneling Times and Superluminality: a Tutorial
}

\author{
Raymond Y. Chiao* \\ Dept. of Physics, Univ. of California \\ Berkeley, CA 94720-7300, U. S. A.
}

January 27,2018

\begin{abstract}
Experiments have shown that individual photons penetrate an optical tunnel barrier with an effective group velocity considerably greater than the vacuum speed of light. The experiments were conducted with a two-photon parametric down-conversion light source, which produced correlated, but random, emissions of photon pairs. The two photons of a given pair were emitted in slightly different directions so that one photon passed through the tunnel barrier, while the other photon passed through the vacuum. The time delay for the tunneling photon relative to its twin was measured by adjusting the path length difference between the two photons in a Hong-Ou-Mandel interferometer, in order to achieve coincidence detection. We found that the photon transit time through the barrier was smaller than the twin photon's transit time through an equal distance in vacuum, indicating that the process of tunneling in quantum mechanics is superluminal. Various conflicting theories of tunneling times are compared with experiment.
\end{abstract}

\section{An introduction to an introduction}

Tunneling, the quantum mechanical process by which a particle can penetrate a classically forbidden region of space, is one of the most mysterious phenomena of quantum mechanics. Yet it is one of the most basic and important processes in Nature, without which we could not even exist, for tunneling is involved in the very first step of the nuclear reaction, $p+p \rightarrow d+e+\overline{\nu_{e}}$, which powers the Sun, the source of energy for life on the Earth.

\footnotetext{
* On appointment as a Miller Research Professor in the Miller Institute for Basic Research in Science.
} 
It may seem that all that we want to know about tunneling is now well understood, given the present mature development of quantum theory. The probability for a particle to penetrate a tunnel barrier is calculated in elementary textbooks on quantum mechanics. Not only has tunneling now been observed in many settings, but it is also the physical basis for many useful devices. However, there remains an open problem concerning the duration of the tunneling process, i.e., the question "How long does is take for the particle to tunnel across the barrier?" This question is still the subject of much controversy, since numerous theories contradict each other in their predictions for "the" tunneling time. Indeed, some of these theories, most notably Wigner's, predict that this time should be superluminal, but others predict that it should be subluminal. Apart from its fundamental interest, a correct solution of this problem is important for determining the speed of devices which are based on tunneling.

Therefore we decided that it would be useful to perform experiments which used the photon as the tunneling particle to measure this time. In this endeavor, we learned the important lesson that a clear operational definition of the experimental method by which the tunneling time is measured is necessary before the above question can even be well formulated. In fact, different operational procedures will lead to conflicting experimental outcomes, so that the time or duration of a process in quantum physics, such as tunneling, is no longer unique, in contrast to the situation in classical physics.

\section{A brief history of tunneling}

Shortly after the advent of the Schrödinger equation, Hund [i] first noticed the possibility of the phenomenon of tunneling, then known as "barrier penetration." He first came across this uniquely quantum phenomenon in a calculation of the splitting of the ground state in a double-well potential, such as in the ammonia molecule in an ammonia maser (to give a modern example). Closely following upon the heels of Hund, Nordheim [2] also applied the then-recently discovered Schrödinger equation to the calculation of the reflection coefficient of an electron from various kinds of interfaces. For the case of a rectangular potential barrier, he noted the remarkable fact that an electron whose energy was insufficient to go over the barrier classically, can still tunnel through the barrier quantum mechanically, whereas classically it would of course always be completely reflected from the barrier. Thus Nordheim extended the case of tunneling between bound states first noticed by Hund to the case of tunneling between continuum states. It is with this latter case that we shall be concerned with here when we examine the problem of tunneling times.

In a rapid sequence of developments in 1928, quantum tunneling was applied both to nuclear physics and to solid state physics, i.e., to an explanation of $\alpha$ radioactive decay of nuclei, and to an explanation of field emission of electrons from a metallic surface. As a prelude to these important develop- 
ments, Oppenheimer [3] performed a correct (but unilluminating) calculation of the rate of ionization of a hydrogen atom by an external field. Following this, Gamow [4], and independently, Gurney and Condon [5] applied the newly understood phenomenon of tunneling to explain the enormous range of $\alpha$-decay rates of radioactive nuclei. In an important application to solid state physics, Fowler and Nordheim [6] performed a tunneling calculation for the rate of emission of electrons into the vacuum due to an intense electric field applied to the surface of a metal, then commonly known as "field emission." In 1934 Zener [7] applied the idea of tunneling to the breakdown of insulators, which was in turn applied to Zener diodes.

After the second World War, various striking new tunneling phenomena were discovered, and new devices based on tunneling were invented. In 1957 Esaki invented the tunnel diode, in which the tunneling of electrons and holes based on internal field emission across a narrow depletion layer in a heavily doped germanium $p$ - $n$ junction led to an anomalous I-V characteristic for the junction: There appeared a negative resistance region in its I-V curve. Due to the separation of opposite-signed charges on opposite sides of the junction, a large internal electric field appeared across the junction. This resulted in a bending of the conduction and valence bands of the semiconductor in the depletion region. The tunneling of electrons and holes across this region accounted for the initial increase of current as the bias voltage across the junction was initially increased from zero. However, as this voltage was increased to a value comparable to the band gap of germanium, the tunneling current decreased. This was due to the decrease in the density of permissible final states for the electrons and holes when they ended up inside the band gap. There resulted a decrease in transmitted current across the junction on account of the Bragg reflection of the electron and holes from the periodic germanium lattice, when their momenta approached that of the lattice. Hence an anomalous negative resistance region appeared in the I-V curve, which allowed the construction of high-frequency electronic oscillators based on the tunnel diode.

In 1962 Josephson predicted the existence of a tunneling supercurrent which traversed a gap separating two superconductors. This superconducting tunnel effect was confirmed experimentally by Giaever and others in Josephson junctions consisting of superconducting thin films separated by a thin oxide barrier. In 1982 Binnig and Rohrer applied electron tunneling to the invention of the scanning tunneling microscope. By monitoring the tunneling current between a sharp metallic needle (etched so that a single atom resides at its tip) and a sample surface as the position of the needle was scanned over the surface, an image of the surface, where individual atoms were resolved, was made possible. Earlier the field-emission microscope invented by Mueller had also allowed the imaging of individual atoms near the tip of an etched metallic needle by imaging the tunneling electrons which had escaped due to field emission from the tip onto a distant screen, but this earlier microscope had only a limited usefulness. 


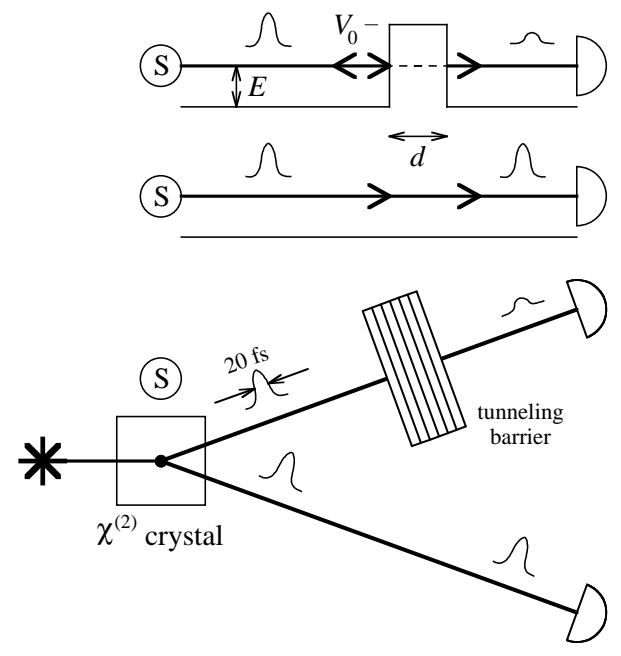

Figure 1: (Top): Gedankenexperiment to measure the tunneling time by means of two particles simultaneously emitted from a source $\mathrm{S}$ and detected by two equidistant detectors. (Bottom): Realization by a spontaneous parametric down-conversion source $\mathrm{S}$, in which a parent photon decays into two daughter photons.

\section{Tunneling time experiments at Berkeley}

The many manifestations of tunneling and the many applications to devices strongly motivated us to examine the unsolved tunneling time problem experimentally. It is very important in attacking this problem to state precisely at the outset the operational definition of the quantity being measured. For the tunneling time for our experiments, this definition is based on the following Gedankenexperiment (see Fig. 1). Suppose that a single parent particle (a photon) decays into two daughter particles (two photons), as in a radioactive decay. Suppose further that these two daughter particles have the same speed in the vacuum (i.e., $c$ in our case), and that they were detected by means of two detectors placed at equal distances from the point of decay. There result simultaneous clicks at the two detectors, e.g., two Geiger counters, which could then be registered in a coincidence counter. Now suppose that we place a tunnel barrier in the path of one of the daughter photons. (The other daughter photon continues to travel unimpeded through the vacuum.) Of course, this would greatly diminish the coincidence count rate. However, whenever a tunneling event does occur, the difference in the time of arrival of the two daughters, as measured by the difference in the time of the clicks of the two detectors, constitutes a precise definition for the tunneling time.

The question concerning the superluminality of the tunneling process can 
now also be precisely stated. Does the click of the detector which registers the arrival of the photon which traversed the tunnel barrier go off earlier or later (on the average) than the click of the detector which registers the arrival the photon which traversed the vacuum? If the tunnel barrier had simply been a thin piece of transparent glass, then the answer would obviously be "later," since the group velocity for a photon inside the glass would be less than the speed of light, and the group delay for the photon traversing the glass relative to that of the vacuum would be positive. However, if, as some tunneling-time theories predict, the tunneling process is superluminal, then the counterintuitive answer would be "earlier," since the effective group velocity for a photon inside the tunnel barrier would be greater than the speed of light, and the group delay for the photon traversing the barrier relative to that of the vacuum would be negative. Hence it is the sign of the relative time between the clicks in the two detectors which determines whether tunneling is subluminal or superluminal.

The reader may ask why relativistic causality is not violated by the superluminality of the tunneling process, if it should indeed be superluminal. It has been shown [8] that special relativity does not forbid the group velocity to be faster than $c$; only Sommerfeld's front velocity must not exceed $c$. Also remember that due to the uncertainty principle the time of emission of the signal photon is not under the experimenter's control.

Presently, the best detectors for photons have picosecond-scale response times, which are still not fast enough to detect the femtosecond-scale time differences expected in our tunneling-time experiment. Hence it was necessary to utilize a Hong-Ou-Mandel interferometer, which has a femtosecond-scale temporal resolution for measuring the time difference between the travel times of the two photons traversing the two arms of the interferometer. By placing the tunnel barrier in one of these arms, a precise measurement of the delay due to tunneling could then be performed.

The tunnel barrier used in our experiments was a dielectric mirror in which periodic layers of alternately high and low index media produce a photonic band gap at the first Brillouin zone edge. The problem of photon propagation in this periodic structure is analogous to that of the Kronig-Penney model for electrons propagating inside a crystal. In particular, near the midgap point on the first Brillouin zone edge, there exists due to Bragg reflection inside the periodic structure an evanescent (i.e., exponential) decay of the transmitted wave amplitude, which is equivalent to tunneling. Note that this Bragg reflection effect is completely analogous to the one occurring in the Esaki tunnel diode mentioned above. One important feature of this kind of tunnel barrier is the fact that it is nondispersive near midgap, and therefore there is little distortion of the tunneling wave packet. 


\section{Tunneling Time Theories}

Another strong motivation for performing experiments to measure the tunneling time was the fact that there were many conflicting theories for this time (see the reviews by Hauge and Støvneng [9], by Landauer and Martin [10], and by Chiao and Steinberg [8]). It suffices here to list the three main contenders:

(1) The Wigner time (i.e., "phase time" or "group delay").

(2) The Büttiker-Landauer time (i.e., "semiclassical time").

(3) The Larmor time (with Büttiker's modification).

The Wigner time calculates how long it takes for the peak of a wave packet to emerge from the exit face of the tunnel barrier relative to the time the peak of the incident wave packet arrives at the entrance face. Since the peak of the wave packet in the Born interpretation is the point of highest probability for a click to occur (see the above Gedankenexperiment), it is natural to expect this to be the relevant time for our experiments. This calculation is based on an asymptotic treatment of tunneling as a scattering problem, and utilizes the method of stationary phase to calculate the position of the peak of a wave packet. The result is simple: this tunneling time is the derivative of the phase of the tunneling amplitude with respect to the energy of the particle.

The Büttiker-Landauer time is based on a different Gedankenexperiment. Suppose that the height of the tunnel barrier is perturbed sinusoidally in time. If the frequency of the perturbation is very low, the tunneling particle will see the instantaneous height of the barrier, and the transmission probability will adiabatically follow the perturbation. However, as one increases the frequency of the perturbation, at some characteristic frequency the tunneling probability will no longer be able to adiabatically follow the rapidly varying perturbation. It is natural to define the tunneling time as the inverse of this characteristic frequency. The result is again simple: for opaque barriers, this tunneling time is the distance traversed by the particle (i.e., the barrier width $d$ ) divided by the absolute value of the velocity of the particle $|v|$. (In the classically forbidden region of the barrier, this velocity is imaginary, but its characteristic size is given by the absolute value).

The Larmor time is based on yet another Gedankenexperiment. Suppose that the tunneling particle had a spin magnetic moment (e.g., the electron). Suppose further that a magnetic field were applied to region of the barrier, but only to that region. Then the angle of precession of the spin of the tunneling particle is a natural measure of the tunneling time. However, Büttiker noticed that in addition to this Larmor precession effect, there is a considerable tendency for the spin to align itself either along or against the direction of the magnetic field during tunneling, since the energy for these two spin orientations is different. The total angular change of the tunneling particle's spin divided by the Larmor precession frequency is Büttiker's Larmor time.

One consequence of the Wigner time is the Hartman effect: The tunneling time saturates for opaque barriers, and approaches for large $d$ a limiting value 
given by the uncertainty principle, $\hbar /\left(V_{0}-E\right)$. The apparent superluminality of tunneling is a consequence of this effect, since as $d$ is increased, there is a point beyond which the saturated value of the tunneling time is exceeded by the vacuum traversal time $d / c$, and the particle appears to have tunneled faster than light.

By contrast, the Büttiker-Landauer theory predicts a tunneling time which increases linearly with $d$ for opaque barriers, as one would expect classically. For a rectangular barrier with a height $V_{0}<<m c^{2}$, the effective velocity $|v|$ is always less than $c$. However, for the periodic structure which we used in our experiment, the effective velocity $|v|$ at midgap is infinite, which is a behavior even more superluminal than that predicted by the Wigner time. This fact makes it easy to distinguish experimentally between these two theories of the tunneling time. However, we hasten to add that the Büttiker-Landauer time may not apply to our experimental situation, as the Gedankenexperiment on which it is based is quite different from the one relevant to our experiment.

Büttiker's Larmor time predicts a tunneling time which is independent of $d$ for thin barriers, but which asymptotically approaches a linear dependence on $d$ in the opaque barrier limit, where it coincides with the Büttiker-Landauer time. In our first experiment it was impossible to distinguish experimentally between this time and the Wigner time. Only in our second experiment could these two theories be clearly distinguished from one another.

\section{Details of the Berkeley Experiments}

Spontaneous parametric down-conversion was the light source used in our experiments 11, 12. An ultraviolet (UV) beam from an argon laser operating at a wavelength of $351 \mathrm{~nm}$ was incident on a crystal of potassium dihydrogen phosphate (KDP), which has a $\chi^{(2)}$ nonlinearity. During the process of parametric down-conversion inside the crystal, a rainbow of many colors was generated in conical emissions around the ultraviolet laser beam, in which one parent UV photon broke up into two daughter photons, conserving energy and momentum. The KDP crystal was cut with an optic axis oriented so that the two degenerate (i.e., equal energy) daughter photons at a wavelength of $702 \mathrm{~nm}$ emerged at a small angle relative to each other. We used two pinholes to select out these two degenerate photons. The size of these pinholes determined the bandwidth of the light which passed through them, and the resulting single-photon wavepackets had temporal widths around 20 fs and a bandwidth of around 6 $\mathrm{nm}$ in wavelength.

The tunnel barrier consisted of a dielectric mirror with eleven quarterwavelength layers of alternately high index material (titanium oxide with $n=$ 2.22 ) and low index material (fused silica with $n=1.45$ ). The total thickness of the eleven layers was $1.1 \mu \mathrm{m}$. This implied an in vacuo traversal time across the structure of 3.6 fs. Viewed as a photonic bandgap medium, this periodic 
structure had a lower band edge located at a wavelength of $800 \mathrm{~nm}$ and an upper band edge at $600 \mathrm{~nm}$. The transmission coefficient of the two photons which were tuned near midgap $(700 \mathrm{~nm})$ was $1 \%$. Since the transmission had a broad minimum at midgap compared to the wave packet bandwidth, there was little pulse distortion. The Wigner theory predicted at midgap a tunneling delay time of around $2 \mathrm{fs}$, or an effective tunneling velocity of $1.8 \mathrm{c}$. The Büttiker-Landauer theory predicted at midgap an infinite effective tunneling velocity, which implies a zero tunneling time.

To achieve the femtosecond-scale temporal resolutions necessary for measuring the tiny time delays associated with tunneling, we brought together these two photons by means of two mirrors, so that they impinged simultaneously at a beam splitter before they were detected in coincidence by two Geiger-mode silicon avalanche photodiodes. There resulted a narrow null in the coincidence count rate as a function of the relative delay between the two photons, a destructive interference effect first observed by Hong, Ou, and Mandel [13. The narrowness of this coincidence minimum, combined with a good signal-to-noise ratio, allowed a measurement of the relative delay between the two photons to a precision of $\pm 0.2 \mathrm{fs}$.

A simple way to understand this two-photon interference is to apply Feynman's rules for the interference of indistinguishable processes. Consider two photons impinging simultaneously on a 50/50 beam splitter followed by two detectors in coincidence detection. When two simultaneous clicks occur at the two detectors, it is impossible even in principle to tell whether both photons were reflected by the beam splitter or whether both photons were transmitted through the beam splitter. In this case, Feynman's rules tell us to add the probability amplitudes for these two indistinguishable process, and then take the absolute square to find the probability. Thus the probability of a coincidence count to occur is given by $\left|r^{2}+t^{2}\right|^{2}$, where $r$ is the complex reflection amplitude for one photon to be reflected, and $t$ is the complex transmission amplitude for one photon to be transmitted. For a lossless beam splitter, time-reversal symmetry leads to the relation $t= \pm i r$. Substituting this into the expression for the coincidence probability, and using the fact that $|r|=|t|$ for a 50/50 beam splitter, we find that this probability vanishes. Thus the two photons must always pair off in the same (random) direction towards only one of the two detectors, an effect which arises from the bosonic nature of the photons.

A schematic of the apparatus we used to measure the tunneling time is given in Fig. 2. The delay between the two daughter photons was adjustable by means of the "trombone prism" mounted on a Burleigh inchworm system, and was measured by means of a Heidenhein encoder with a $0.1 \mu \mathrm{m}$ resolution. A positive sign of the delay due to a piece of glass was determined as corresponding to a motion of the prism towards the glass. The multilayer coating of the dielectric mirror (i.e., the tunnel barrier) was evaporated on only half of the glass mirror substrate. This allowed us to translate the mirror so that the beam path passed either through the tunnel barrier in an actual measurement of the tunneling 


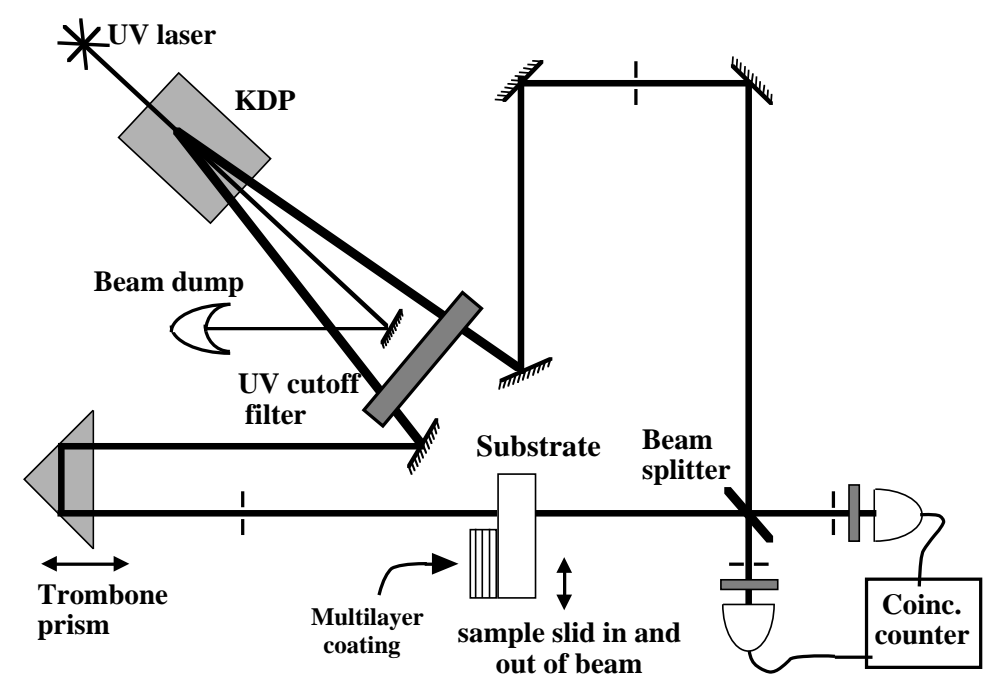

Figure 2: Schematic of the Berkeley experiments to measure the tunneling time.

time, or through the uncoated half of the substrate in a control experiment. In this way, one could obtain data with and without the barrier in the beam, i.e., a direct comparison between the delay through the tunneling barrier and the delay for traversing an equal distance in air. The normalized data obtained in this fashion is shown in Fig. 3(a), with the barrier oriented at normal incidence $\left(\theta=0^{\circ}\right)$. Note that the coincidence minimum with the tunnel barrier in the beam is shifted to a negative value of delay relative to that without the barrier in the beam. This negative shift indicates that the tunneling delay is superluminal.

To double-check the sign of this shift, which is crucial for the interpretation of superluminality, we tilted the mirror towards Brewster's angle for the substrate $\left(\theta=56^{\circ}\right)$, where there is a very broad minimum in the reflection coefficient as a function of angle. Near Brewster's angle this minimum is so broad that it is not very sensitive to the difference between the high and low indices of the successive layers of dielectrics. Thus to a good approximation, the reflections from all layers vanish simultaneously near this angle. Hence the Bragg reflection responsible for the band gap disappears, and the evanescent wave behavior and the tunneling behavior seen near normal incidence disappears. The dielectric mirror should then behave like a thin piece of transparent glass with a positive delay time relative to that of the vacuum. Detailed calculations not using the above approximations also show that at $\theta=55^{\circ}$, the sign of the shift should indeed revert to its normal positive value.

The data taken in p-polarization at $\theta=55^{\circ}$ is shown in Fig. 3(b). The reversal of the sign of the shift is clearly seen. Therefore one is confronted with 

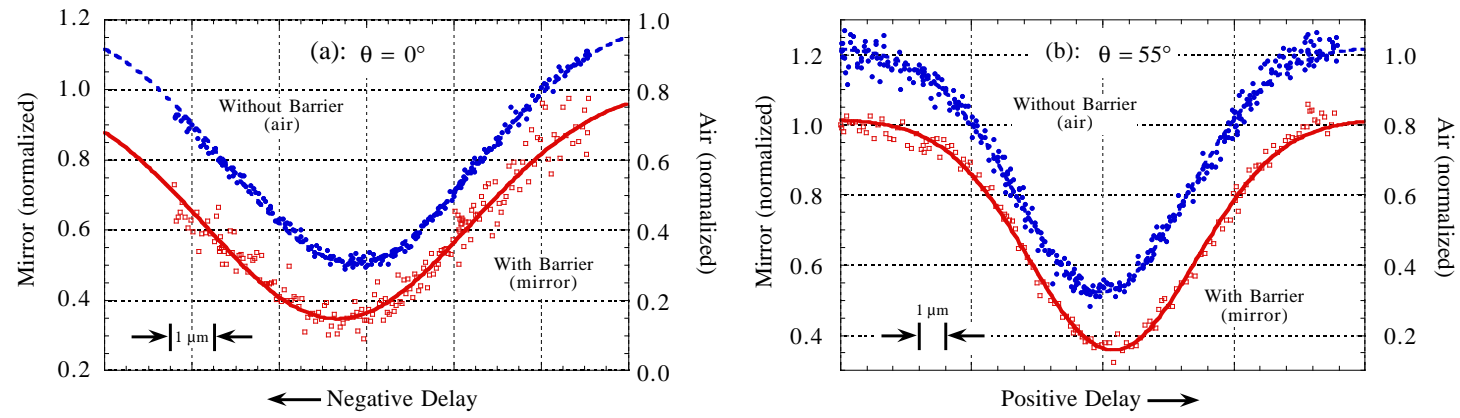

Figure 3: (a) Coincidence rate vs delay (i.e., the position of the trombone prism in Fig. 2) with and without the tunnel barrier (mirror) in the beam path at normal incidence. (b) The same with the mirror tilted towards Brewster's angle.

a choice of the data either in Fig. 3(a) or in Fig. 3(b) as showing a superluminal shift. Since we know that the delay in normal dielectrics as represented by Fig. 3(b) should be subluminal, this implies that the tunneling delay in Fig. 3(a) should be superluminal. Therefore the data in Fig. 3(a) implies that after traversing the tunnel barrier, the peak of a photon wave packet arrived $1.47 \pm$ $0.21 \mathrm{fs}$ earlier than it would had it traversed only vacuum.

Another reason for tilting the mirror is that one can thereby distinguish between the Wigner time and Büttiker's Larmor time, as they differ considerably in the region near the band edge, which occurs near Brewster's angle. This can be seen in Fig. 4, where there is a considerable divergence as the band edge is approached between the solid line representing the theoretical prediction of the Wigner time, and the long-dashed line representing that of Büttiker's Larmor time. The data points in Fig. 4 seem to rule out Büttiker's Larmor time (although again we hasten to add that this theory may not apply to our experiment). The agreement with Wigner's theory is better, but there are discrepancies which are not understood.

Other experiments confirming the superluminality of tunneling have been performed in Cologne, Florence, and Vienna [14, 15, 16]. The Cologne and Florence groups performed microwave experiments, and the Vienna group performed a femtosecond laser experiment. All these groups have confirmed the Hartman effect. One of these groups 17 has claimed to have sent Mozart's 40th symphony at a speed of $4.7 c$ through a microwave tunnel barrier $114 \mathrm{~mm}$ long consisting of a periodic dielectric structure similar to our dielectric mirror. However, the further implication that their experiment represents a violation of causality is in our opinion unfounded [8].

Recently, an experiment indicating the simultaneous existence of two differ- 


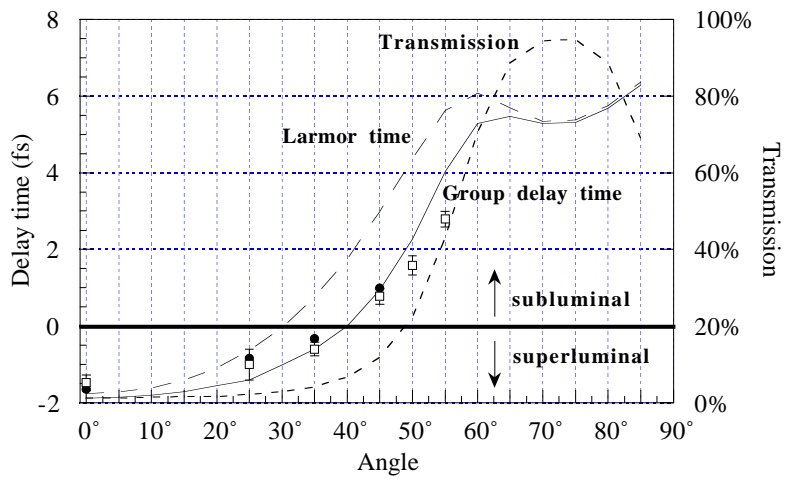

Figure 4: Temporal shifts of the minima seen in data such as in Fig. 3 plotted as a function of incidence angle, compared with the theoretical predictions of the Wigner time (solid curve) and of Büttiker's Larmor time (long-dashed curve). The transmission vs incidence angle (short-dashed line). All curves are for ppolarization. Solid circles and open squares denote data from two different tunnel barrier samples.

ent tunneling times was performed in Rennes [18]. In frustrated total reflection (FTIR), the tunneling of photons through an air gap occurs between two glass prisms when a light beam is incident upon this gap beyond the critical angle. The Rennes group observed in FTIR both a lateral displacement of the tunneling beam of light and an angular deflection of this beam. These two effects could be interpreted as evidence for two different tunneling times that simultaneously occurred in the same tunneling barrier. The lateral displacement is related to the Wigner time, and the angular deflection is related to the Büttiker-Landauer time. As evidence for this, they cited the saturation of the beam displacement (the Hartman effect), and the linear increase of the beam deflection, as the gap was increased.

\section{Conclusions}

The experiments at Berkeley and elsewhere thus indicate that the tunneling process is superluminal. In our opinion, this does not imply that one can communicate faster than $c$, despite claims to the contrary by Heitmann and Nimtz [17]. The group velocity cannot be identified as the signal velocity of special relativity, by which a cause is connected to its effect. Rather, it is Sommerfeld's front velocity which exclusively plays this role. However, even if one were to define the group velocity as a "signal" velocity, no causal loop paradoxes can arise [19].

Although the controversies amongst the various tunneling theories have not yet been fully resolved by experiment, a good beginning has been made in this 
direction. In particular, it is now clear that one cannot rule out the Wigner time simply on the grounds that it yields a superluminal tunneling time. It also appears that there may exist more than one tunneling time. Hopefully, the mysterious role of time in quantum mechanics will be elucidated by these studies.

\section{Acknowledgments}

I would like to thank Prof. Rodolfo Bonifacio for inviting me to give the opening lecture of this conference on the mysteries, puzzles, and paradoxes of quantum physics. I would also like to thank Dr. Paul Kwiat and Prof. Aephraim Steinberg for their collaboration on the Berkeley experiments, and for many helpful discussions. This work was supported by the ONR under Grant No. N000149610034.

\section{References}

[1] F. Hund, Zeitschrift für Physik 43 (1927) 805.

[2] L. Nordheim, Zeitschrift für Physik 46 (1927) 833.

[3] J. R. Oppenheimer, Phys. Rev. 31 (1928) 66; Proc. Nat. Acad. Sci. 14 (1928) 363.

[4] G. Gamow, Zeitschrift für Physik 51 (1928) 204; 52 (1928) 510.

[5] R. W. Gurney and E. U. Condon, Nature 122 (1928) 439; Phys. Rev. 33 (1929) 127.

[6] R. H. Fowler and L. Nordheim, Proc. Roy. Soc. (London) A119 (1928) 173.

[7] C. Zener, Proc. Roy. Soc. (London) 145 (1934) 523.

[8] R. Y. Chiao and A. M. Steinberg, in Progress in Optics XXXVII, E. Wolf, ed., (Elsevier, Amsterdam, 1997), p. 345.

[9] E. H. Hauge and J. A. Støvneng, Rev. Mod. Phys. 61 (1989) 917.

[10] R. Landauer and Th. Martin, Rev. Mod. Phys. 66 (1994) 217.

[11] A. M. Steinberg, P. G. Kwiat, and R. Y. Chiao, Phys. Rev. Lett. 71(1993) 708.

[12] A. M. Steinberg, and R. Y. Chiao, Phys. Rev. 51(1995) 3525.

[13] C. K. Hong, Z. Y. Ou and L. Mandel, Phys. Rev. Lett. 59 (1987) 2044.

[14] A. Enders and G. Nimtz, J. Phys. I France 3 (1993) 1089. 
[15] A. Ranfagni, P. Fabeni, G.P. Pazzi and D. Mugnai, Phys. Rev. E 48(1993) 1453.

[16] Ch. Spielmann, R. Szipöcs, A. Stingl and F. Krausz, Phys. Rev. Lett. 73(1994) 2308.

[17] W. Heitmann and G. Nimtz, Phys. Lett. A 196(1994) 154.

[18] Ph. Balcou and L. Dutriaux, Phys. Rev. Lett. 78(1997) 851.

[19] J. C. Garrison, M. W. Mitchell, R. Y. Chiao, and E. L. Bolda, Phys. Lett. A 245 (1998) 19. 